\title{
Factores Biológicos que Determinan la Competencia de Commelina erecta con otras MALEzas en Sistemas de Cultivo ${ }^{1}$
}

\author{
Biological Factors Determining Commelina erecta Competition with other Weeds in Cultivated \\ Systems
}

NISENSOHN, L. ${ }^{2}$, TUESCA, D. ${ }^{2}$, FACCINI, D. ${ }^{2}$, PURICELLI, E. ${ }^{3}$ y VITTA, J. ${ }^{4+}$

\begin{abstract}
RESUMEN - Se estudió la competencia de Commelina erecta originada de semilla y de rizoma con Digitaria sanguinalis y Amaranthus quitensis. Los objetivos del trabajo fueron: 1) estudiar la dinámica del crecimiento de plantas de estas malezas en mezclas binarias y en monoculturas; 2) estimar el rendimiento relativo de cada especie (RR) y el rendimiento relativo total (RRT) de las distintas mezclas y 3) utilizar un modelo dinámico para analizar la importancia de distintos atributos específicos sobre el resultado de la competencia. El crecimiento de las monoculturas de todas las especies ajustó a un modelo sigmoide. Los RR de las distintas malezas variaron en función de la especie acompañante, de la fecha de muestreo y del año. La competencia fue parcial (RRT > 1) únicamente en mezclas que incluyeron plantas de C. erecta originadas de rizomas. A partir de las simulaciones con el modelo, se determinó que el resultado de la competencia depende del tamaño inicial (BI) de rizomas de C. erecta, por otro lado, el análisis de sensibilidad señala la gravitación de la tasa intrínseca de crecimiento ( $\mathrm{r}$ ) en la definición de RR. Bajo condiciones de competencia, los parámetros biológicos que definieron el crecimiento de las especies estudiadas fueron especialmente $\mathrm{r}$ y BI. Los resultados destacan la compleja interacción de factores que determinan el balance competitivo, tanto entre ambas formas de propagación de C. erecta, como entre ellas y otras malezas.
\end{abstract}

Palabras-clave: modelos de competencia, Commelina erecta, Digitaria sanguinalis, Amaranthus quitensis.

\begin{abstract}
Competition of C. erecta from seed and rhizome origin with Digitaria sanguinalis and Amaranthus quitensis was studied. The objectives of the study were: 1) determine growth dynamics of plants of these weed species in binary mixtures and in monocultures; 2) estimate the relative yield of each species (RY) and relative yield total (RYT) of the different mixtures, and 3) to use a dynamic model to analyze the importance of different specific attributes on the result of the competition. The growth of the monoculture of all species fitted to a sigmoidal model. The RY of the different species varied relative to the accompanying species, the sampling date and the year. Competition was partial (RYT > 1) only in mixtures including $\mathbf{C}$. erecta plants originated from rhizomes. From the simulations with the model it was determined that the result of the competition depends on the initial size (BI) of C. erecta rhizomes. Furthermore, the sensibility analysis highlights the importance of the intrinsic growth rate ( $r$ ) in the definition of RY. Under competition conditions the biological parameters defining the growth of the species studied were particularly $r$ and BI. The results emphasize the complex interaction of the factors determining the competitive balance, between both propagation forms of C. erecta, and between those forms and other weed species.
\end{abstract}

Keywords: competition models, Commelina erecta, Digitaria sanguinalis, Amaranthus quitensis.

1 Recebido para publicação em 6.10.2010 e na forma revisada em 18.2.2011.

2 Cátedra de Malezas, Facultad de Ciencias Agrarias, Universidad Nacional de Rosario, CC 14 (S 2125 ZAA), Zavalla, Santa Fe - Argentina, Tel: 0341-970080, lnisen@arnet.com. ar, ${ }^{3}$ Cátedra de Terapéutica Vegetal, Facultad de Ciencias Agrarias, Universidad Nacional de Rosario, CC 14 (S 2125 ZAA), Zavalla, Santa Fe-Argentina; ${ }^{4}$ Fallecido en el año 2006. 


\section{INTRODUCCIÓN}

La estructura y la productividad de las comunidades vegetales esta muy influenciada por las relaciones competitivas entre sus componentes (Grace \& Tilman, 1990). Esto es válido tanto para los sistemas naturales como para los agroecosistemas. Se puede definir la competencia como una interacción entre individuos, producida por la demanda común de un recurso limitado y que conduce a la reducción en la supervivencia, crecimiento y/ o reproducción de por lo menos uno de los competidores (Begon et al., 1996).

La competencia es un fenómeno complejo, definido por múltiples factores. Su resultado es altamente dependiente de la tasa de crecimiento inicial de cada especie (Kropff \& van Laar, 1993). Dicho crecimiento está, a su vez, influenciado por el momento relativo de emergencia y el tamaño inicial de los órganos de propagación (Holt \& Orcutt, 1991).

Se han propuesto numerosos indices para cuantificar la competencia (Snaydon, 1991). Todos ellos relacionan la producción de biomasa de cada especie creciendo en monocultura con la de las especies creciendo en la mezcla. Uno de dichos índices es el "rendimiento relativo total" (RRT) (Wit, 1960), el cual mide el grado en que las especies se complementan en el uso de los recursos. Otro de los indices empleados se ha definido como "agresividad" (McGilchrist \& Trenbath, 1971) y es una medida de la habilidad competitiva relativa de cada una de las especies. En la literatura, se citan otros índices utilizados, cuya interpretación biológica, en muchos casos, no difiere sustancialmente de la de los previamente mencionados (Snaydon, 1991). Cousens (1996) sostiene que todos los indices basados en la acumulación de biomasa hasta un cierto punto del ciclo de crecimiento no son buenos estimadores de la intensidad de la competencia. Propone como alternativa hacer hincapié en la dinámica de la competencia en el tiempo, para lo cual recurre a la estimación del rendimiento relativo de cada especie (RR), el cual relaciona la biomasa específica en la mezcla con la de la monocultura.

Existen pocos estudios que intentan promover, a partir de la estimación de la habilidad competitiva, una mayor comprensión de las causas por las que una maleza resulta más competitiva que otra (Roush \& Radosevich, 1985; Wilson \& Wright, 1990; Holt \& Orcutt, 1991). En Argentina, se ha determinado la disminución en los rendimientos del cultivo de soja (Abascal et al., 2002) debido al efecto de Commelina erecta, pero no existen estudios relativos a su habilidad competitiva y a la de otras especies de malezas. Este tipo de información es útil a la hora de interpretar la importancia de $C$. erecta en las actuales comunidades de malezas de la región sojera núcleo.

El uso de modelos matemáticos constituye una herramienta valiosa para el estudio de la competencia entre cultivos y malezas (Kropff \& van Laar, 1993). Un modelo matemático se define como una ecuación o una serie de ecuaciones que representan el comportamiento del sistema (France \& Thornley, 1984). La ventaja de la modelización es que ella no sólo permite describir o comprender un sistema en circunstancias ya observadas sino que también permite predecir su comportamiento en situaciones desconocidas (Vitta, 1994).

El presente trabajo tiene como objetivos 1) Estudiar la dinámica del crecimiento de plantas de $C$. erecta originadas de semillas y de rizomas, de Amaranthus quitensis y de Digitaria sanguinalis, tanto en mezclas binarias como en monoculturas; 2) Determinar indices destinados a cuantificar el grado de competencia de las distintas mezclas y la habilidad competitiva de cada uno de sus componentes; y 3) Utilizar el modelo dinámico (COMPITE) basado en el trabajo de Satorre (1995) para analizar la importancia de distintos atributos especificos sobre el resultado de la competencia.

\section{MATERIALES Y MÉTODOS}

Los experimentos se llevaron a cabo durante los años 2001 y 2002. Los tratamientos consistieron en monoculturas de C. erecta, provenientes tanto de semillas como de rizomas, $D$. sanguinalis y A. quitensis. Además, fueron sembradas mezclas binarias que combinaron cada una de las formas de reproducción de $C$. erecta entre sí y con las otras especies. Se emplearon macetas de 
plástico de 7 L de capacidad, completadas con una mezcla de dos partes de tierra y una de perlita. El diseño del experimento fue aditivo estándar (Cousens, 1991), ya que incluyó monoculturas con una misma densidad de plantas (una planta por maceta) y una sola mezcla binaria en la cual la densidad de cada uno de los componentes era similar a la de las monoculturas. De esta manera, la densidad total de la mezcla fue el doble del de las respectivas monoculturas (es decir, dos plantas por maceta). Este tipo de diseño es apropiado para un estudio inicial de la interacción entre especies, debido a que el factor de interés es el tipo de vecino y no la densidad o la proporción de la mezcla (Cousens, 1991). E1 diseño experimental fue completamente aleatorizado con 24 repeticiones. Las macetas se regaron diariamente y fueron tratadas con insecticidas y funguicidas cuando era necesario. En ambos años, la siembra se realizó a fines de enero y la emergencia de plantas tuvo lugar durante la primera semana de febrero y fue simultánea para todas las especies. Aproximadamente cada diez días se cortaban plantas de tres macetas de cada uno de los tratamientos y se determinaba su peso seco. Los ensayos tuvieron una duración aproximada de sesenta dias y se realizaron en condiciones semicontroladas.

Para describir la dinámica del crecimiento de las especies en monocultura, los datos obtenidos se ajustaron a la siguiente ecuación logística:

$$
B=\frac{K}{1+\exp ^{(b-r t)}}
$$

donde $B$ es la biomasa de la especie $\left(\mathrm{g} \mathrm{m}^{-2}\right)$ y $t$ es el tiempo. $K, b$ y $r$ son parámetros de la ecuación. $K\left(\mathrm{~g} \mathrm{~m}^{-2}\right)$ es la biomasa máxima y $r$ es la tasa intrínseca de crecimiento de la especie (g g día ${ }^{-1}$ ).

Para cada fecha de extracción, se cuantificó la habilidad competitiva relativa de cada una de las especies, a través del cálculo del rendimiento relativo (RR) de cada especie, de acuerdo a la siguiente fórmula:

$$
R=B_{a b} / B_{a a}
$$

donde $B_{a b}$ es la biomasa de la especie a en la mezcla con la especie $b$ y $B_{a a}$ es la biomasa de la especie a en monocultura. En los casos en que no hubiera competencia, las especies crecerian igual en la mezcla que en la monocultura, por lo que el RR alcanzaría valores iguales a uno. En competencia, los valores de $R R$ serían menores que uno, hasta alcanzar valores de cero en el caso que la especie en la mezcla no sobreviviera a la interacción. De esa manera, se puede considerar RR como un valor indicativo de la habilidad competitiva de una especie en una mezcla (Cousens, 1996).

Se estimó también el rendimiento relativo total (RRT) de las mezclas:

$$
R R T=\left(B_{a b} / B_{a a}\right)+\left(B_{b a} / B_{b b}\right) \quad \text { [ec. 3] }
$$

donde $B_{b a}$ es la biomasa de la especie $b$ en la mezcla con la especie $a$ y $B_{b b}$ es la biomasa de la especie $\mathrm{b}$ en monocultura. Un valor de $R R T=$ 1 indica que la competencia es plena (es decir, que las especies son mutuamente excluyentes). En cambio, en los casos en que el RRT es mayor que 1, existe complementariedad en el uso de los recursos y las especies compiten sólo parcialmente, mientras que un valor de $R R T=2$ implica ausencia de competencia. (Snaydon, 1991).

\section{Descripción y aplicación del modelo COMPITE}

E1 modelo describe la competencia entre dos especies a partir del crecimiento logístico de la biomasa de cada una en monocultura (ecuación 1), desde el momento de la emergencia de1 $50 \%$ de las plántulas.

Para simular el crecimiento de la especie, la tasa de crecimiento de la especie en cualquier momento del ciclo es obtenida derivándose la ecuación (1):

$$
\Delta B / \Delta t=B[r(K-B) / K]
$$

Es decir, la tasa de crecimiento es función de la biomasa acumulada hasta ese momento. En competencia, la tasa de crecimiento de cada especie depende de la biomasa total de la mezcla. De allí que la ecuación (4) se modifica de la siguiente manera:

$$
\begin{array}{r}
\Delta B_{a} / \Delta t=B_{a} \cdot\left[r_{a}\left(K_{a}-\left(\left(b_{a} / K_{a}\right)+B_{b} / K_{b}\right)\right) K_{a}\right] / K_{a} \\
{[\text { ec. 5] }}
\end{array}
$$


$\mathrm{y}$

$\Delta B b / \Delta t=B b \cdot[r b(K b-((b b / K b)+B a / K a)) K b] / K b$

[ec. 6]

donde los subindices $a$ y $b$ representan a cada una de las especies en la mezcla.

Las variables de entrada que requiere el modelo para realizar las simulaciones se obtienen a partir del ajuste del crecimiento de la biomasa de las monoculturas a la ecuación (1), y son las siguientes:

I. La biomasa inicial $(B I)$, calculada aplicando la ecuación (1) para $t=0$, es decir, la biomasa por unidad de área al momento de la emergencia.

II. La biomasa máxima de cada especie $(K)$ en la monocultura.

III. La tasa intrínseca de crecimiento $(r)$ de las especies en monocultura.

El modelo supone que el recurso capturado por un componente se transforma en biomasa $\mathrm{y}$, de esta manera, no está disponible para el otro componente de la mezcla. E1 modelo asume que en la mezcla la competencia es completa (es decir, RRT = 1).

\section{Aplicación del modelo}

El modelo fue empleado para simular el efecto de distintos valores iniciales de biomasa de rizomas de $C$. erecta sobre su rendimiento relativo en las distintas mezclas.

Además, se realizó un análisis de sensibilidad para determinar el efecto de modificaciones de las distintas variables de entrada (BI, K y r) obtenidas en 2002 sobre el rendimiento relativo de cada especie en las distintas mezclas. Para ello, se incrementó en un 10\% cada una de las variables de entrada, mientras que el resto permanecía constante. La sensibilidad relativa de la variable $p\left(S_{p}\right)$ fue calculada de acuerdo a la siguiente fórmula:

$$
S_{p}=\frac{\Delta R R / R R}{0,1}
$$

donde $\triangle R R$ es la variación del rendimiento relativo debida a un cambio en la variable $p$, $R R$ es el rendimiento relativo previo a dicho cambio, y 0,1 representa la proporción relativa de cambio de $p$.

\section{Análisis estadístico}

Tanto los parámetros de la ecuación (1) como los valores finales de RR fueron comparados mediante pruebas de $t(\mathrm{p}=0,05)$. Los valores de RRT de las mezclas fueron sometidos a un análisis de la variancia y se realizó un test de LSD $(\mathrm{P}=0,05)$ para comparar los valores obtenidos con un RRT $=1$. Para la validación del modelo COMPITE, se ajustó una regresión lineal entre los valores finales de biomasa observados y simulados para las distintas mezclas. La pendiente de dicha regresión se comparó mediante una prueba de $t(p=0,05)$ con una pendiente teórica de uno, la cual supone una correspondencia plena entre ambas variables.

\section{RESULTADOS Y DISCUSIÓN}

En los dos años, el crecimiento de las monoculturas de todas las especies ajustó a un modelo sigmoideo. En 2002, las plantas de C. erecta de rizomas acumularon biomasa más rápido que las de semillas, mientras que en 2001 las tendencias fueron más semejantes. El crecimiento de $A$. quitensis en ambos años tuvo por lo general valores intermedios entre las dos formas de propagación de C. erecta. La mayor biomasa de $D$. sanguinalis se debió al crecimiento superior de esta especie en la segunda mitad de los experimentos (Figura 1). En ambos años, las plantas de $D$. sanguinalis alcanzaron valores de biomasa máxima $(\mathrm{K})$ estadísticamente superiores al del resto de las monoculturas. En 2001, el valor de K de C. erecta de rizomas fue significativamente mayor que el de $C$. erecta de semillas y el de A. quitensis. En ninguno de los años, la tasa intrínseca de crecimiento $(\mathrm{r}$ ) difirió significativamente entre las monoculturas. La biomasa inicial (BI) de todas las especies fue menor en el año 2001 que en el 2002. En el primer año, el valor de BI de C. erecta de rizomas superó al del resto de las monoculturas entre 6 y 43 veces, mientras que en el segundo año estuvo entre 11 y 105 veces (Tabla 1 ).

E1 rendimiento relativo total (RRT), durante el año 2001, fue significativamente superior 
a uno en la mezcla de plantas de $C$. erecta de semillas y de rizomas. En el año 2002, el RRT fue mayor que uno en todas las mezclas que incluían plantas de $C$. erecta de rizomas. Esto significa que la competencia fue parcial, manifestándose complementariedad de recursos entre los componentes de la mezcla (Tabla 2). Esto podría explicarse al considerar que las raíces de las plantas provenientes de semillas se encuentran en menor cantidad y más superficiales que las plantas provenientes de rizomas, y podrían explorar, por lo tanto, diferentes profundidades del suelo (Wilson, 1988). Estudios previos de competencia realizados en macetas, sugieren que la competencia entre raíces generalmente es más importante que la competencia entre tallos (Wilson, 1988; Satorre \& Snaydon, 1992).

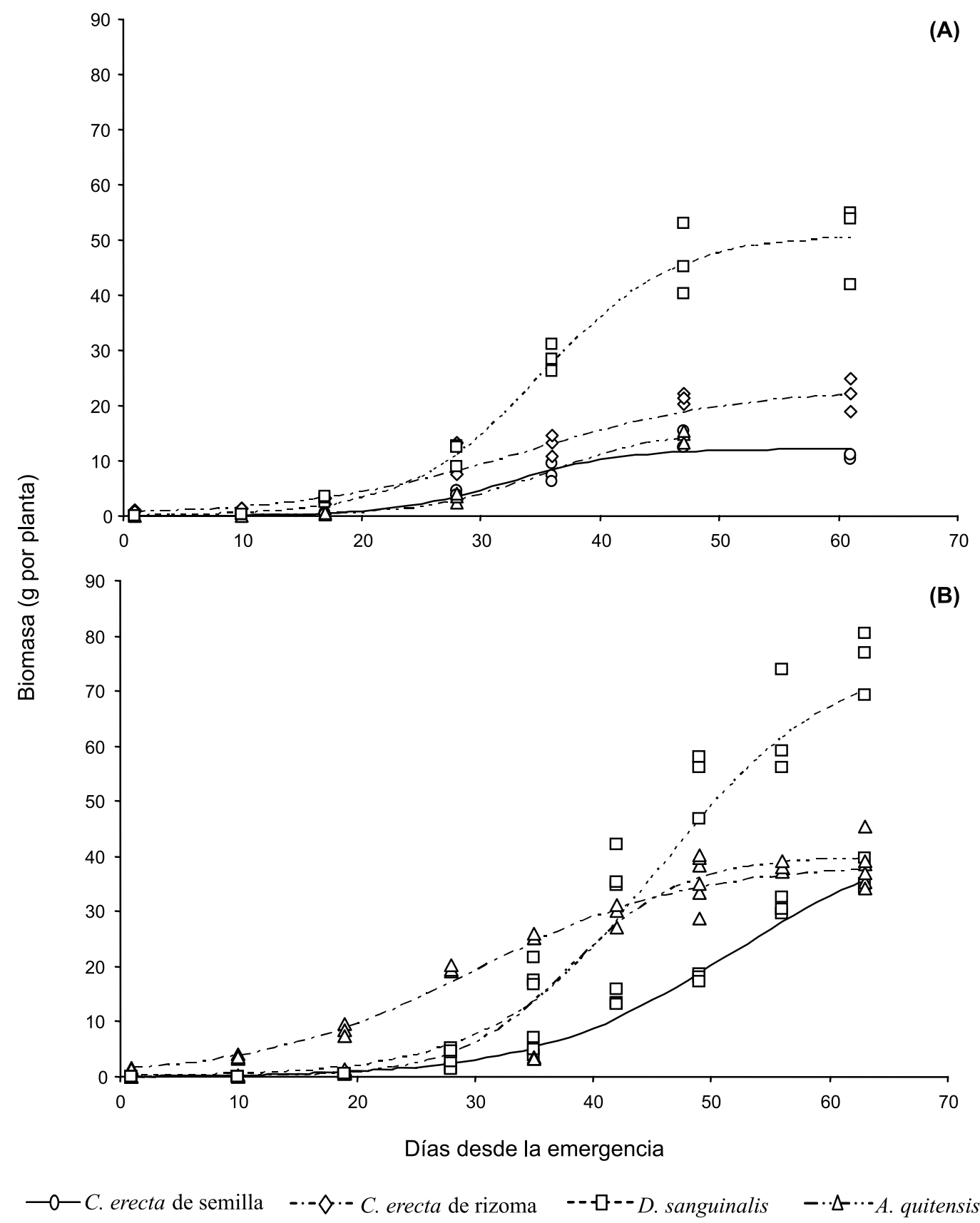

Figura 1 - Dinámica de crecimiento de las especies en monoculturas - (A) 2001 y (B) 2002. 
Tabla 1 - Variables de entrada del modelo COMPITE

\begin{tabular}{|l|c|c|c|c|c|c|c|c|}
\hline & \multicolumn{2}{|c|}{ C. erecta $(\mathrm{s})$} & \multicolumn{2}{c|}{ C. erecta $(\mathrm{r})$} & \multicolumn{2}{c|}{ D. sanguinalis } & \multicolumn{2}{c|}{ A. quitensis } \\
\cline { 2 - 10 } & 2001 & 2002 & 2001 & 2002 & 2001 & 2002 & 2001 & 2002 \\
\hline \multirow{2}{*}{$\mathrm{K}$} & 12,13 & 44,29 & 23,04 & 38,58 & 50,82 & 76,24 & 15,54 & 39,75 \\
& $(0,63)$ & $(3,35)$ & $(1,39)$ & $(0,82)$ & $(1,98)$ & $(3,53)$ & $(1,39)$ & $(2,11)$ \\
\hline \multirow{2}{*}{$\mathrm{r}$} & 0,21 & 0,12 & 0,11 & 0,11 & 0,18 & 0,14 & 0,2 & 0,21 \\
& $(0,05)$ & $(0,01)$ & $(0,02)$ & $(0,008)$ & $(0,02)$ & $(0,01)$ & $(0,07)$ & $(0,06)$ \\
\hline BI & 0,014 & 0,089 & 0,556 & 1,359 & 0,092 & 0,125 & 0,003 & 0,013 \\
\hline CMR & 1,68 & 3,19 & 4,29 & 2,48 & 11,89 & 19,65 & 0,32 & 11,04 \\
\hline
\end{tabular}

$K\left(\mathrm{~g} \mathrm{~m}^{-2}\right)=$ biomasa máxima, $r\left(\mathrm{~g} \mathrm{~g} \mathrm{día}^{-1}\right)=$ tasa intrínseca de crecimiento, $\mathrm{BI}\left(\mathrm{g} \mathrm{m}^{-2}\right)=$ biomasa inicial y $\mathrm{CMR}=$ cuadrado medio residual. $(\mathrm{r})$ indica plantas de C. erecta que provienen de rizoma y (s) que provienen de semilla_ Entre paréntesis se indican los errores estándar.

Tabla 2 - Rendimiento relativo total (RRT) de las mezclas en los dos años de experiencias

\begin{tabular}{|c|c|c|c|c|c|}
\hline Año & $\begin{array}{c}\text { C. erecta (s) } \\
+ \\
\text { A. quitensis }\end{array}$ & $\begin{array}{c}\text { C. erecta (r) } \\
+ \\
\text { A. quitensis }\end{array}$ & $\begin{array}{l}\text { C. erecta (s) } \\
+ \\
\text { C. } \operatorname{erecta}(\mathrm{r})\end{array}$ & $\begin{array}{c}\text { C. erecta (s) } \\
+ \\
\text { D. sanguinalis }\end{array}$ & $\begin{array}{c}\text { C. erecta (r) } \\
+ \\
\text { D. sanguinalis }\end{array}$ \\
\hline 2001 & 0,9520 & & 1,3712 & 1,2653 & 1,1326 \\
\hline 2002 & 1,0180 & 1,2658 & 1,0944 & 0,9985 & 1,1870 \\
\hline
\end{tabular}

$(\mathrm{r})$ indica plantas de C. erecta que provienen de rizoma y ( $\mathrm{s}$ ) que provienen de semilla. El asterisco indica diferencias significativas de un valor hipotético de uno $(\mathrm{p}=0,05)$

Los rendimientos relativos $(\mathrm{RR})$ de las distintas plantas en competencia variaron en función de la especie acompañante, de la fecha de muestreo y del año (Figura 2). La habilidad competitiva de una especie está asociada positivamente con una emergencia temprana y un crecimiento inicial rápido que anticipa la captación de los recursos limitantes (Kropff, 1988). Sin embargo, en algunos casos, especies que retrasan su emergencia o tienen una tasa de crecimiento inicial lenta, pueden, más tarde, tornarse dominantes en una mezcla (Cousens, 1991; Satorre \& Snaydon, 1992). En los experimentos aquí presentados, se manifestaron ambas alternativas. En algunas de las mezclas, el resultado de la competencia fue independiente del período de crecimiento considerado. Por ejemplo, en 2002, los valores de RR de $C$. erecta de rizomas se mantuvieron siempre cercanos a uno, indicando que su producción en la monocultura fue similar a la de la mezcla, mientras que los del otro componente de la mezcla disminuyeron progresivamente hasta valores próximos a cero. En cambio, en otras mezclas, hubo un cambio en la habilidad competitiva con el tiempo. En 2001, el RR final de C. erecta de rizoma no difirió del de $D$. sanguinalis, y sólo superó marginalmente $(\mathrm{p}=0,10)$ al de $C$. erecta de semilla. $D$. sanguinalis inicialmente tendió a disminuir sus valores de RR, pero al promediar el experimento se revirtió esa tendencia y finalmente su RR no difirió del de la especie acompañante. Ese incremento en la habilidad competitiva de $D$. sanguinalis, con el tiempo, se corresponde con su mayor crecimiento en monocultura en la etapa final del experimento. En dicho año, no se presentó el gráfico de $C$. erecta de semilla y A. quitensis por mortalidad de las plantas (Figura 2). Los cambios en la habilidad competitiva en función del momento del ciclo de crecimiento podrían estar vinculados con la dinámica del desarrollo morfológico de las especies. Hay mucha evidencia circunstancial que sugiere que el tiempo relativo del desarrollo morfológico es crítico para el resultado de la competencia (Cousens et al., 1991).

E1 resultado de la competencia también dependió del año. En el caso de las plantas de C. erecta provenientes de rizomas, su RR final superó al de las demás en 2002, pero no en 2001 (Figura 2). Esta habilidad competitiva 
(A)
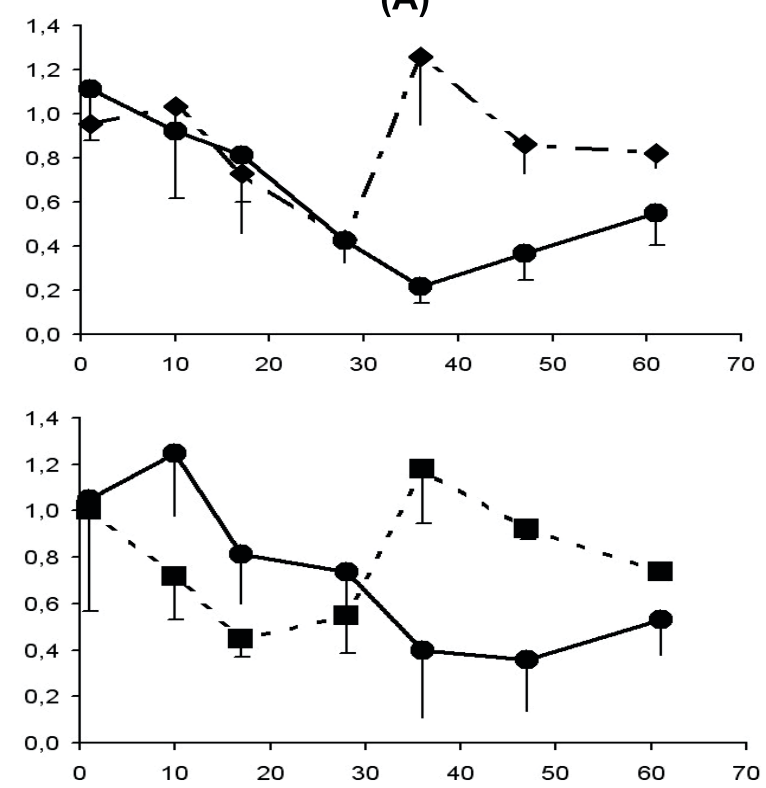

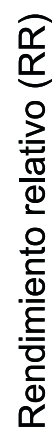
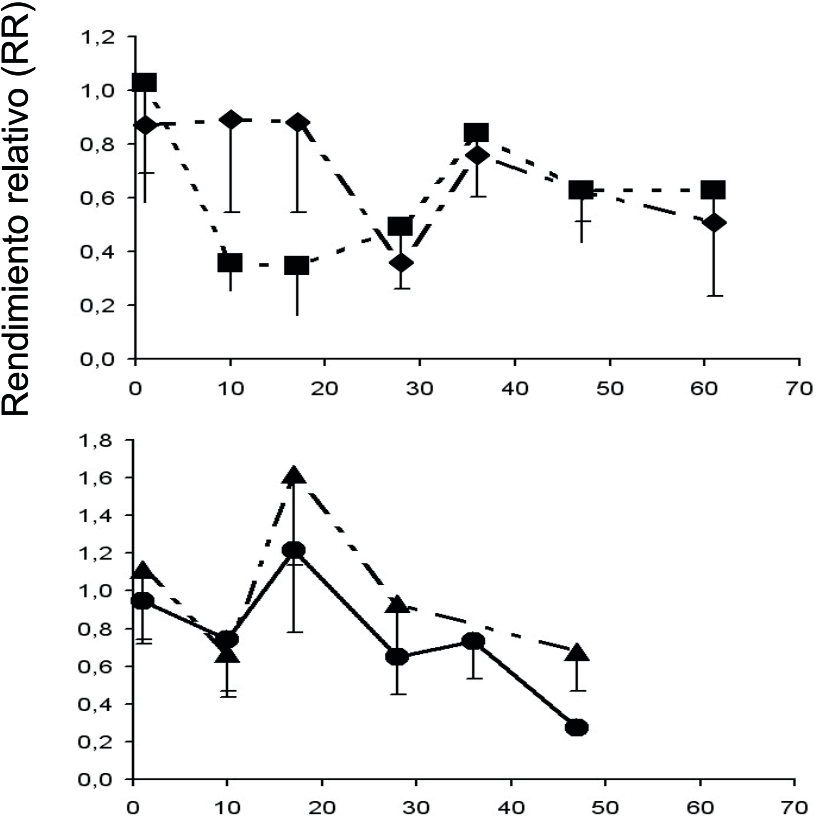

C. erecta de semilla

-.- C. erecta de rizomas

-- -- D. sanguinalis

$-\ldots$ - . A. quitensis

Las barras representan el desvío estándar
(B)
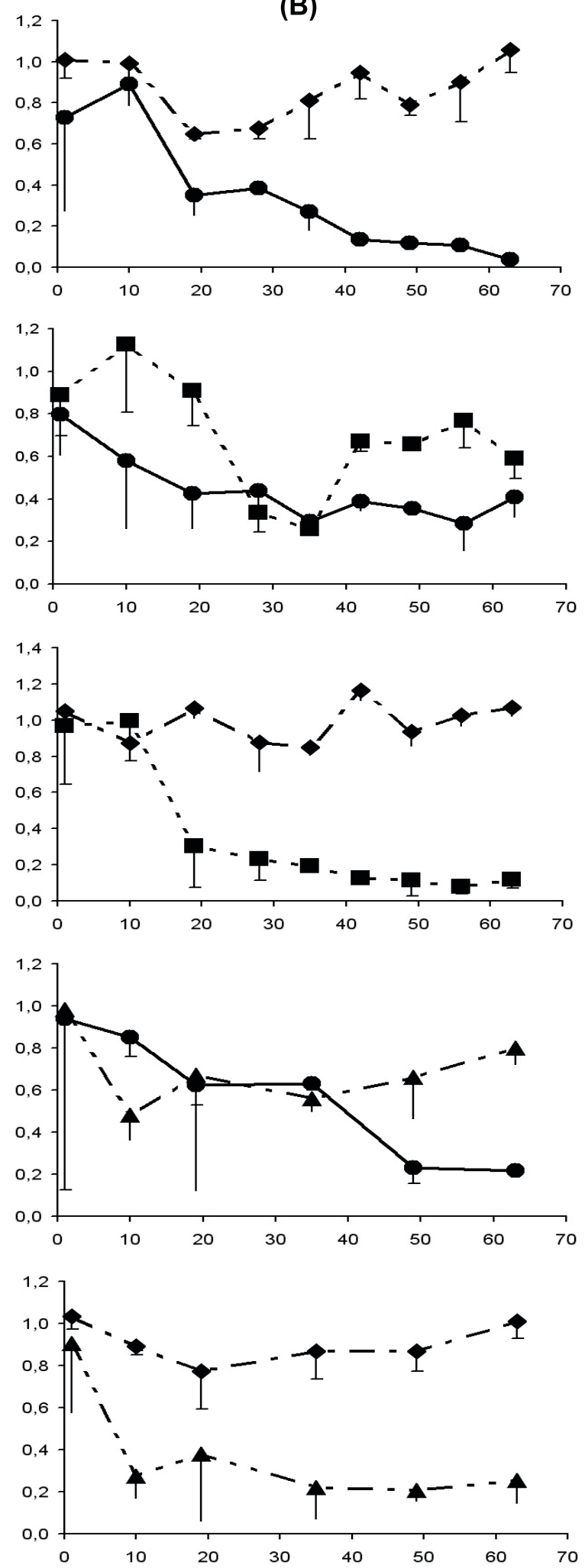

Días desde la emergencia

Figura 2 - Rendimiento relativo (RR) de las especies creciendo en mezclas - (A) 2001 y (B) 2002. 
diferente de $C$. erecta de rizomas según al año podría estar asociada con su valor de biomasa inicial, el cual fue relativamente mayor en 2002 que en 2001 (Tabla 1).

La habilidad competitiva de $C$. erecta con otras especies estuvo vinculada con su forma de propagación. Los valores de RR de $C$. erecta de semilla tendieron a disminuir con el tiempo en la mayoría de los casos, alcanzando valores finales que oscilaron entre 0,04 y 0,5. Dependiendo del año, al final del experimento, la habilidad competitiva de $C$. erecta de rizomas fue igual o superior al de las otras especies. En cambio, C. erecta de semillas fue igual competidora que $D$. sanguinalis, pero menos competidora que $A$. quitensis (Figura 2). Este hecho también se puede explicar a través de las diferencias en los valores de BI (Tabla 1).

La capacidad del modelo COMPITE para simular la biomasa final de las especies en competencia fue evaluada a través de la relación entre los valores de biomasa observados y los simulados por el modelo. Considerando los datos de ambos años, la regresión entre ambas variables fue significativa $\left(R^{2}=0,84\right.$; $\left.\mathrm{p}=4,43375 \times 10^{-8}\right)$ y su pendiente no difirió significativamente de uno (Figura 3 ).

Mediante sucesivas corridas del modelo, fue posible simular el efecto de BI de $C$. erecta de rizomas sobre su valor de RR en competencia con $C$. erecta de semillas, $D$. sanguinalis y A. quitensis. Se observó que con valores de BI de $1,3 \mathrm{~g}$ (coincidente con el valor de BI de C. erecta de rizomas en 2002) el rendimiento relativo de $C$. erecta de rizomas estuvo próximo a 0,9 . Con valores inferiores, $R R$ tendió a disminuir hasta alcanzar valores entre 0,6 y 0,8 con valores de BI de 0,4 g. En las simulaciones realizadas, se puso de manifiesto la relación positiva entre la habilidad competitiva y el tamaño inicial de rizomas (Figura 4) y coincide además con experimentos previos de competencia con malezas perennes (Holt \& Orcutt, 1991).

El análisis de sensibilidad mostró que en todas las mezclas, el valor de RR fue más sensible a cambios en $\mathrm{r}$ que a cambios en $\mathrm{K}$ o en BI. A su vez, los individuos de C. erecta originados de semillas resultaron más sensibles a cambios en las variables de entrada que los originados de rizomas. Aumentos en el 10\% de los valores de $\mathrm{r}$ de $C$. erecta se correspondieron con valores de sensibilidad entre 0,165 y 0,394 para plantas originadas de rizomas y entre 1,312 y 1,920 para plantas de semillas. Los valores de RR de A. quitensis $\mathrm{y}$ $D$. sanguinalis fueron por lo general más sensibles a modificaciones en todas las variables de entrada que los de $C$. erecta de rizomas, pero menos sensibles que los de C. erecta de semillas (Tabla 3).

El análisis de sensibilidad señala la gravitación de la tasa intrínseca de crecimiento $(\mathrm{r})$ en la definición de RR. De esta manera, incluso pequeñas variaciones en el valor de $r$ afectarian significativamente el resultado de

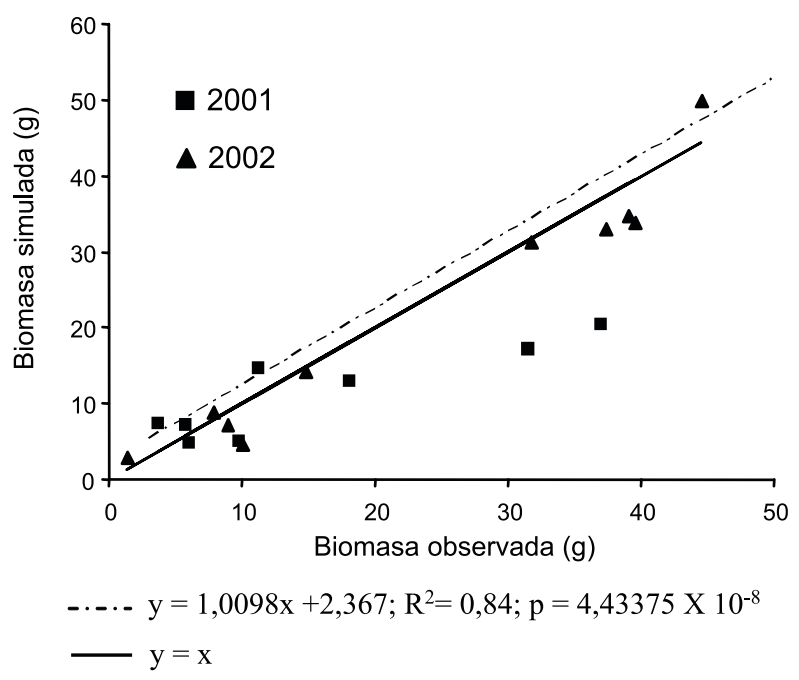

Figura 3 - Relación entre los valores simulados y observados de biomasa de las especies en competencia.

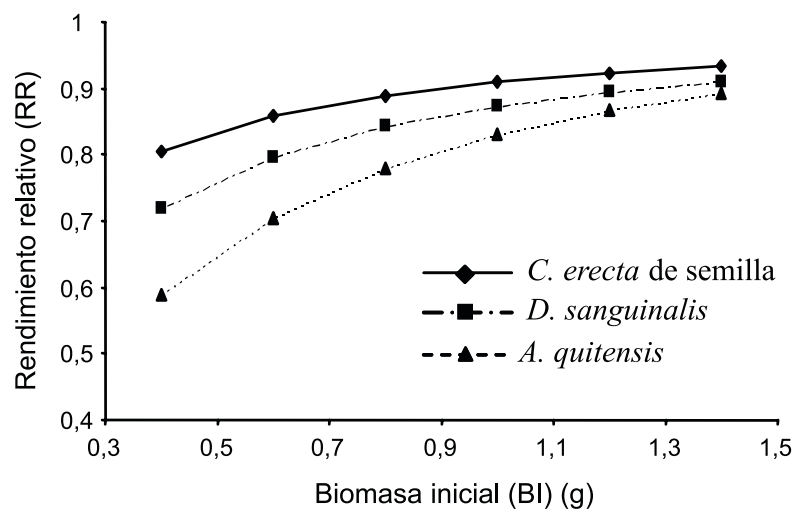

Figura 4 - Simulación del efecto de la biomasa inicial (BI) de $C$. erecta de rizomas sobre su rendimiento relativo (RR). 
Tabla 3 - Sensibilidad relativa del rendimiento relativo (RR) de las especies a aumentos del 10\% en las variables de entrada del modelo

\begin{tabular}{|c|c|c|c|}
\hline Especie & & C. $\operatorname{erecta}(\mathrm{r})$ & C. erecta (s) \\
\hline \multirow{3}{*}{ C. erecta (s) } & $\mathrm{r}$ & $-0,248$ & 1,312 \\
\hline & $\mathrm{K}$ & 0,061 & $-0,523$ \\
\hline & $\mathrm{Bi}$ & $-0,066$ & 0,572 \\
\hline \multirow{3}{*}{ C. $\operatorname{erecta}(\mathrm{r})$} & $\mathrm{r}$ & 0,165 & 2,396 \\
\hline & $\mathrm{K}$ & $-0,069$ & 0,966 \\
\hline & $\mathrm{Bi}$ & 0,062 & $-0,878$ \\
\hline & & D. sanguinalis & C. erecta (s) \\
\hline \multirow{3}{*}{ C. erecta (s) } & $\mathrm{r}$ & $-1,595$ & 1,402 \\
\hline & $\mathrm{K}$ & 0,106 & $-0,113$ \\
\hline & $\mathrm{Bi}$ & $-0,325$ & 0,343 \\
\hline \multirow{3}{*}{ D. sanguinalis } & $\mathrm{r}$ & 1,115 & $-2,421$ \\
\hline & $\mathrm{K}$ & $-0,232$ & 0,481 \\
\hline & $\mathrm{Bi}$ & 0,230 & $-0,479$ \\
\hline & & D. sanguinalis & C. $\operatorname{erecta}(\mathrm{r})$ \\
\hline \multirow{3}{*}{ C. $\operatorname{erecta}(\mathrm{r})$} & $\mathrm{r}$ & $-2,628$ & 0,249 \\
\hline & $\mathrm{K}$ & 1,067 & $-0,105$ \\
\hline & $\mathrm{Bi}$ & $-0,978$ & 0,095 \\
\hline \multirow{3}{*}{ D. sanguinalis } & $\mathrm{r}$ & 2,664 & $-0,381$ \\
\hline & $\mathrm{K}$ & $-0,655$ & 0,081 \\
\hline & $\mathrm{Bi}$ & 0,709 & $-0,088$ \\
\hline & & A. quitensis & C. erecta (s) \\
\hline \multirow{3}{*}{ C. erecta (s) } & $\mathrm{r}$ & $-1,100$ & 1,920 \\
\hline & $\mathrm{K}$ & 0,244 & $-0,469$ \\
\hline & $\mathrm{Bi}$ & $-0,22$ & 0,499 \\
\hline \multirow{3}{*}{ A. quitensis } & $\mathrm{r}$ & 0,782 & $-2,869$ \\
\hline & $\mathrm{K}$ & $-0,135$ & 0,486 \\
\hline & $\mathrm{Bi}$ & 0,129 & $-0,469$ \\
\hline & & A. quitensis & C. $\operatorname{erecta}(\mathrm{r})$ \\
\hline \multirow{3}{*}{ C. $\operatorname{erecta}(\mathrm{r})$} & $\mathrm{r}$ & 3,424 & 0,394 \\
\hline & $\mathrm{K}$ & 1,439 & $-0,172$ \\
\hline & $\mathrm{Bi}$ & $-1,290$ & 0,156 \\
\hline \multirow{3}{*}{ A. quitensis } & $\mathrm{r}$ & 4,954 & $-0,633$ \\
\hline & $\mathrm{K}$ & $-0,727$ & 0,094 \\
\hline & $\mathrm{Bi}$ & 0,777 & $-0,10$ \\
\hline
\end{tabular}

$\mathrm{k}\left(\mathrm{g} \mathrm{m}^{-2}\right)=$ biomasa máxima, $\mathrm{r}\left(\mathrm{g} \mathrm{g} \mathrm{día}^{1}\right)=$ tasa intrínseca de crecimiento y BI $\left(\mathrm{g} \mathrm{m}^{-2}\right)=$ biomasa inicial. (r) indica plantas de C. erecta provienen de rizoma y (s) que provienen de semilla.

la competencia. Por el contrario, el impacto que tuvieron tanto la biomasa inicial (BI) como la biomasa máxima $(\mathrm{K})$ fue mucho menos importante. Vitta \& Satorre (1999) emplearon e1 modelo COMPITE para analizar la competencia de soja con Datura ferox y A. quitensis y llegaron a conclusiones similares a las aquí obtenidas. E1 valor de $\mathrm{r}$ es indicativo de la velocidad de crecimiento inicial de la especie y puede estar afectado por distintos métodos de control como, por ejemplo, el uso de un herbicida. En ese caso, si la eficacia del herbicida es diferente para cada una de las especies de la mezcla, su empleo podría afectar las relaciones de dominancia de la mezcla. Al analizar las distintas variables que componen un modelo, no sólo interesa definir cuales son aquellas a la cual el modelo es más sensible, sino que también importa cuales pueden ser modificadas más fácilmente (Cousens \& Mortimer, 1995). En nuestro caso, por ejemplo, BI podría ser fácilmente afectado a través del control químico o cultural de la maleza.

En base a los resultados, se podría indicar que los parámetros biológicos que definen el crecimiento de las especies estudiadas, bajo condiciones de competencia, serian especialmente r y BI. Los resultados obtenidos en este trabajo destacan la compleja interacción de factores que determinan el balance competitivo, tanto entre ambas formas de propagación de C. erecta, como entre ellas con otras malezas. Por otra parte, el modelo COMPITE fue capaz de poner de manifiesto la importancia que tiene en la definición de la competencia, tanto el tamaño inicial de los rizomas como la velocidad inicial del crecimiento de las especies. Toda esta información tiene importancia a la hora de analizar las causas del éxito ecológico de C. erecta en el nuevo modelo productivo regional.

\section{LITERATURA CITADA}

ABASCAL, F.; MORAN LEMIR, A. H.; GARCÍA, A. E. Interferencia de diferentes densidades de Commelina erecta $\mathrm{L}$. sobre los rendimientos de soja (Glycine max L.). Actas XI Jornadas Fitosanitarias. Río Cuarto: 2002.

BEGON, M.; HARPER, J. L.; TOWNSEND, C. R. Ecology: individuals, populations and communities. 3.ed. Oxford: Blackwell Science, 1996. 1068.

COUSENS, R. Aspect of the design and interpretation of competition (interference) experiments. Weed Technol., v. 5 , p. 664-673, 1991.

COUSENS, R. D. et al. Dynamics of competition between wild oats (Avena fatua L.) and winter cereals. Weed Res., v. 31, p. 203-210, 1991 
COUSENS, R.; MORTIMER, M. Dynamics of weed populations. Cambrige: Cambridge University Press, 1995. $332 \mathrm{p}$.

COUSENS, R. Design and interpretation of interference studies: Are some methods totally unacceptable? New Zealand J. For. Sci., v. 26, n. 1, p. 5-18, 1996.

FRANCE, J.; THORNLEY, J. H. M. Mathematical models in agriculture. London: Butterworths, 1984. 350 p.

GRACE, J. B.; TILMAN, D. Perspectives on plant competition. San Diego: Academic Press, 1990. 483 p.

HOLT, J. S.; ORCUTT, D. R. Functional relationships of growth and competitiveness in perennial weeds and cotton (Gossypium hirsutum). Weed Sci., v. 39, p. 575-584, 1991.

KROPFF, M. J. Modelling the effects of weeds on crop production. Weed Res., v. 28, p. 465-471, 1988

KROPFF, M. J.; van LAAR, H. H. (Eds.). Modelling cropweed interactions. Wallingford: $C A B$ International, 1993. $304 \mathrm{p}$

MCGILCHRIST, C. A.; TRENBATH, B. R. A revised analysis of plant competition experiments. Biometrics, v. 27, p. $659-671,1971$.

ROUSH, M. L.; RADOSEVICH, S. R. Relationships between growth and competitionships of four annual weeds. J. Applied Ecol., v. 22, p. 895-905, 1985.
SATORRE, E. H. Simulación dinámica de la competencia entre cereales invernales y malezas a partir de atributos de las especies en monoculturas. In: CONGRESO LATINOAMERICANO DE MALEZAS, 12., 1995, Montevideo. Actas... Montevideo: 1995. p. 221-227.

SATORRE, E. H.; SNAYDON, R. W. A comparison of root and shoot competition between spring cereals and Avena fatua L. Weed Res., v. 32, p. 45-55, 1992.

SNAYDON, R. W. Replacement or additive designs for competition studies? J. Applied Ecol., v. 28, p. 930-946, 1991.

VITTA, J. I.; SATORRE, E. Validation of a dynamic model of weed-crop competition. Weed Res., v. 39, p. 259-269, 1999.

VITTA, J. I. Empleo de modelos matemáticos para la predicción de la competencia entre trigo (Triticum aestivum) y Sinapis alba. 1994. 108 f. (Tesis Doctoral) Universidad Politécnica de Madrid. E.T.S.I. Agrónomos, 1994.

WILSON, B. J. Shoot competition and root competition. J. Applied Ecol., v. 25, p. 279-296, 1988.

WILSON, B. J.; WRIGHT, K. J. Predicting the growth and competitive effects of annual weeds in wheat. Leed Res., v. 30 , p. 201-211, 1990.

WIT, C. T. On competition. Verslagen Landb. Onderzoekingen, v. 66, p. 1-82, 1960. 